\title{
COMMENTARY
}

\section{Epinephrine for prehospital cardiac arrest with non-shockable rhythm}

\author{
Samuel J Stratton ${ }^{1,2}$ \\ See related research by Goto et al., http://ccforum.com/content/17/5/R188
}

\begin{abstract}
Cardiopulmonary arrest research and guidelines have generally focused on the treatment and management of ventricular fibrillation and pulseless ventricular fibrillation (electrical shockable rhythms). Less investigation has been done on the subpopulation of cardiopulmonary arrest victims that present with nonshockable rhythms. In a new paper, Goto, Maeda, and Goto present evidence that early use of epinephrine for treatment is associated with better survival with functional outcome. While there is a lack of evidence to support epinephrine for management of cardiopulmonary arrest presenting with initial shockable rhythms (presumed primary cardiac origin), there is now evidence that epinephrine may potentially benefit those presenting with nonshockable cardiopulmonary arrest (presumed heterogeneous origins). Further research on nonshockable rhythm cardiopulmonary arrest is needed to understand the subpopulation and develop better treatment guidelines.
\end{abstract}

\section{Introduction}

Goto and colleagues [1] are to be congratulated for their contribution to the medical knowledge base regarding the efficacy of epinephrine in resuscitation of nonshockable rhythm cardiac arrest. Epinephrine is commonly used for cardiopulmonary arrest resuscitation [2,3]. Epinephrine for treatment of cardiopulmonary arrest became commonly used more than four decades ago with support of observational and theoretical information rather than structured trials. Theoretically, when

\footnotetext{
Correspondence: strattos@ucla.edu

'University of California, Los Angeles, David Geffen School of Medicine and Fielding School of Public Health, Los Angeles, CA 90095, USA

${ }^{2}$ Emergency Medical Services, Orange County California Health Care Agency, Santa Ana, CA 92701, USA
}

used for cardiac arrest, epinephrine increases systemic vascular resistance with secondary increased coronary blood flow, but there has long been concern that it also causes myocardial dysfunction when used in the arrest setting [4].

In 2004, Stiell and co-authors published a landmark study that questioned the effectiveness of advanced life support interventions when added to a system that had optimized access to cardiopulmonary resuscitation, defibrillation, and rapid transport to advanced hospital care [5]. Included in the advanced life support interventions was use of epinephrine. Hagihara and co-authors showed in a study published in 2012 [6] that the use of epinephrine for cardiopulmonary arrest was associated with return of spontaneous circulation but negatively was associated with decreased survival and functional outcome.

The current paper by Goto, Maeda, and Goto [1] is an interesting contradiction to current thought and literature regarding use of epinephrine for out-of-hospital cardiac arrest. As illustrated in the literature referenced in the above paragraph, there is doubt that epinephrine use during resuscitation has benefit in survival and functional outcome. In their study, Goto and co-authors found an association between improved survival and functional outcome and the use of epinephrine for treatment of cardiopulmonary arrest presenting in other than the rhythms of ventricular fibrillation or pulseless ventricular tachycardia, which can immediately be treated with electrical counter shock. It should be emphasized that the outcome measures used by Goto and coauthors were actual survival and functional outcome, not return of spontaneous circulation or other pseudoindicators often used by resuscitation researchers.

The data presented by Goto and co-authors support the use of epinephrine for cardiopulmonary arrest presenting with non-shockable rhythms. This commentary explores the implications these findings have for future resuscitation and research. 


\section{Discussion}

Traditional research on cardiopulmonary arrest has focused on the general state of cardiopulmonary arrest. Recognizing that cardiopulmonary arrest can be of primary cardiac origin and secondary to other acute conditions, many researchers now limit their study populations to those with ventricular fibrillation and pulseless ventricular tachycardia in an effort to select subjects with high probability of primary cardiac origin arrest. Little focus has been on those types of arrest that do not present in ventricular fibrillation or pulseless ventricular tachycardia (shockable rhythms). In fact, the term pulseless electrical activity was created as a 'catch all' term for presenting rhythms that were less likely associated with cardiac arrest originating from coronary artery occlusion. Cardiopulmonary arrest presenting with a non-shockable rhythm can have many origins, including septic shock, hypovolemia, anaphylaxis, and acute cerebral events.

Emphasis in resuscitation research has been on the subpopulation presenting with shockable ventricular rhythms, who are known to have better potential to respond to witnessed arrest, bystander cardiopulmonary resuscitation, and early defibrillation. This research emphasis has ignored a significant subgroup of the overall cardiopulmonary arrest population - those presenting with non-shockable rhythm. In fact, more than a decade ago the non-shockable subpopulation was shown to have potential for response to advanced life support resuscitation measures [7]. The current paper by Goto and coauthors supports aggressive resuscitation attempts for those presenting with non-shockable rhythms. Their work alerts the critical care community to the need for more emphasis on research and evaluation of cardiopulmonary arrest victims who present with non-shockable rhythms.

A cardiac emphasis has limited the scope and understanding of cardiopulmonary arrest. A broader understanding of the causes of sudden cardiopulmonary collapse would seem logical. While Goto and co-authors did not explore the etiology of cardiopulmonary arrest in the victims studied, one can assume that those in collapse and profound shock (pulseless) from sepsis, anaphylaxis, and toxicities causing vasodilation would potentially respond favorably to epinephrine. Moreover, if patients in the non-shockable rhythm group show positive responses to the vasoconstrictor epinephrine, some of these are likely hypovolemic and would respond favorably to early intravenous fluid administration. As was done by Goto and co-authors, scientific exploration of the effects of vasoconstrictors among cardiopulmonary arrest victims presenting with non-shockable rhythms is needed. In addition, research is needed to define the most common underlying causes for non- shockable cardiopulmonary arrest so that potential resuscitation strategies can be developed, such as administration of epinephrine and early intravenous fluid bolus.

\section{Conclusion}

Non-shockable cardiopulmonary arrest victims constitute a heterogeneous subgroup that is separate from victims of shockable rhythm cardiopulmonary arrest. For decades clinical resuscitation guidelines and research have focused on shockable rhythm cardiopulmonary arrest. The paper by Goto and co-authors is an important addition to the scientific knowledge base that supports aggressive exploration and development of better resuscitation guidelines that focus on the non-shockable rhythm cardiopulmonary arrest cohort.

\section{Competing interests}

The author declares that he has no competing interests.

Published: 04 Oct 2013

\section{References}

1. Goto Y, Maeda T, Goto YN: Effects of prehospital epinephrine during out-of-hospital cardiac arrest with initial non-shockable rhythm: an observational cohort study. Crit Care 2013, 17:R188.

2. American Heart Association: VF/pulseless VT. In Advanced Cardiac Life Support. Edited by Sinz E, Navarro K. Dallas: American Heart Association; 2011:59-63.

3. Nolan JP, Soar J, Zideman DA, Biarent D, Bossaert LL, Deakin C, Koster RW, Wyllie J, Böttiger B, ERC Guidelines Writing Group: European Resuscitation Council Guidelines for resuscitation 2010 section 1 executive summary. Resuscitation 2010, 81:1232-1234.

4. Tang W, Weil MH, Sun S, Noc M, Yang L, Gazmuri RJ: Epinephrine increases the severity of postresuscitation myocardial dysfunction. Circulation 1995, 92:3089-3093.

5. Stiell IG, Wells GA, Field B, Spaite DW, Nesbitt LP, De Maio VJ, Nichol G, Cousineau D, Blackburn J, Munkley D, Luinstra-Toohey L, Campeau T, Dagnone E, Lyver M, Ontario Prehospital Advanced Life Support Study Group: Advanced cardiac life support in out-of-hospital cardiac arrest. N Engl J Med 2004, 351:647-656.

6. Hagihara A, Hasegawa M, Abe T, Nagata T, Wakata Y, Miyazaki S: Prehospital epinephrine use and survival with out-of-hospital cardiac arrest. JAMA 2012, 307:1161-1168.

7. Stratton SJ, Niemann JT: Outcome from out-of-hospital cardiac arrest caused by nonventricular arrhythmias: contribution of successful resuscitation to overall survivorship supports the current practice of initiating out-of-hospital ACLS. Ann Emerg Med 1998, 32:448-453.

$10.1186 / \mathrm{cc} 13044$

Cite this article as: Stratton: Epinephrine for prehospital cardiac arrest with non-shockable rhythm. Critical Care 2013, 17:1006 